\title{
A bioinspired omniphobic surface coating on medical devices prevents thrombosis and biofouling
}

\section{Citation}

Leslie, Daniel C, Anna Waterhouse, Julia B Berthet, Thomas M Valentin, Alexander L Watters, Abhishek Jain, Philseok Kim, et al. 2014. "A Bioinspired Omniphobic Surface Coating on Medical Devices Prevents Thrombosis and Biofouling." Nature Biotechnology 32 (11) (October 12): 11341140. doi:10.1038/nbt.3020.

\section{Published Version}

doi: $10.1038 / \mathrm{nbt} .3020$

\section{Permanent link}

http://nrs.harvard.edu/urn-3:HUL.InstRepos:34298865

\section{Terms of Use}

This article was downloaded from Harvard University's DASH repository, and is made available under the terms and conditions applicable to Other Posted Material, as set forth at http:// nrs.harvard.edu/urn-3:HUL.InstRepos:dash.current.terms-of-use\#LAA

\section{Share Your Story}

The Harvard community has made this article openly available.

Please share how this access benefits you. Submit a story.

Accessibility 


\section{A bioinspired surface coating for medical devices that}

prevents thrombosis and biofouling

Daniel C. Leslie ${ }^{1,2,6 \dagger}$, Anna Waterhouse $e^{1,3,5 \dagger}$, Julia B. Berthet ${ }^{1,3,5}$, Thomas M.

Valentin $^{1,3}$, Alexander L. Watters ${ }^{1,3}$, Abhishek Jain ${ }^{1,2}$, Philseok Kim ${ }^{1,2}$, Ben D.

Hatton $^{1,2, \ddagger}$, Arthur Nedder ${ }^{4}$, Kathryn Donovan ${ }^{4}$, Elana H. Super ${ }^{1}$, Caitlin

Howell ${ }^{1,2}$, Christopher P. Johnson ${ }^{1,2}$, Thy L. Vu ${ }^{1,2}$, Dana E. Bolgen ${ }^{1}$, Sami

Rifai $^{1}$, Anne Hansen ${ }^{1,5}$, Michael Aizenberg ${ }^{1,2}$, Michael Super ${ }^{1,3,6}$, Joanna

Aizenberg ${ }^{1,2}$, and Donald E. Ingber ${ }^{1,2,3,6 *}$

${ }^{1}$ Wyss Institute for Biologically Inspired Engineering, Harvard University, Boston, MA 02115, USA.

${ }^{2}$ Harvard School of Engineering and Applied Sciences, Cambridge, MA 02138, USA.

${ }^{3}$ Harvard Medical School, Boston, MA 02115, USA

${ }^{4}$ Animal Research, Boston Children's Hospital, Boston MA 02115, USA.

${ }^{5}$ Division of Newborn Medicine, Boston Children's Hospital, Boston, MA 02115, USA.

${ }^{6}$ Vascular Biology Program, Departments of Pathology and Surgery, Boston Children's Hospital, Boston, MA 02115, USA.

${ }^{\dagger}$ Authors contributed equally

${ }^{\ddagger}$ Current address: Department of Materials Science and Engineering, University of Toronto, Ontario, Canada

${ }^{*}$ Correspondence to: $\quad$ Donald E. Ingber, M.D., Ph.D.

Wyss Institute at Harvard University

3 Blackfan Circle, CLSB 5

Boston, MA 02115

Email: don.ingber@wyss.harvard.edu

Tel: 617-432-7044

Fax: $617-432-7068$ 


\section{Summary}

Thrombosis and biofouling of extracorporeal circuits and indwelling medical devices cause significant morbidity and mortality worldwide. We describe a bioinspired coating that repels blood from virtually any material by covalently tethering a molecular layer of perfluorocarbon, which holds a thin liquid film of medical-grade perfluorocarbon on the substrate surface, mimicking the liquid layer certain plants use to prevent adhesion. This coating prevents fibrin attachment, reduces platelet adhesion and activation, suppresses biofilm formation, and is stable under blood flow in vitro. Surface-coated medical-grade tubing and catheters, assembled into arteriovenous shunts and implanted in living pigs, remain patent for at least 8 hours without anticoagulation. This coating technology offers the potential to significantly reduce anticoagulation in patients while preventing thrombotic occlusion and biofouling of medical devices.

Countless lives have been saved by implantable medical devices (e.g., total artificial hearts, ventricular assist devices, pacemakers, cardioverterdefibrillators, and central lines) and extracorporeal devices that flow whole human blood outside the body through indwelling catheters and external circuits, during cardiopulmonary bypass (CPB), hemodialysis, and extracorporeal membrane oxygenation $(E C M O)^{1,2}$. However, the need to co-administer soluble anticoagulant drugs, such as heparin, with many of these procedures, significantly reduces their safety and hampers their effectiveness ${ }^{3,4}$. Without 
systemic anticoagulation, these extracorporeal and indwelling devices can rapidly occlude due to thrombosis because clots form when fibrin and platelets in the flowing blood adhere to the surfaces of these artificial materials ${ }^{5}$. Unfortunately, heparin causes significant morbidity and mortality including post-operative bleeding, thrombocytopenia, hypertriglyceridemia, hyperkalemia and hypersensitivity ${ }^{6}$, and its use is contraindicated in several patient populations ${ }^{7}$. In fact, the majority of drug-related deaths from adverse clinical events in the United States are due to systemic anticoagulation ${ }^{8}$.

This need to prevent blood clotting while minimizing administration of anticoagulant drugs has led to the search for biomaterial surface coatings that can directly suppress blood clot formation. The most successful approach to date has been to chemically immobilize heparin on blood-contacting surfaces to reduce thrombosis and lower anticoagulant administration ${ }^{9,10}$. Although this approach has been widely adopted, major limitations persist because the surface-bound heparin leaches, resulting in a progressive loss of anticoagulation activity $^{4,11}$ and the use of heparin-coated materials has not led to a drastic reduction in the clinical use of soluble heparin ${ }^{12}$. Some high-flow dialysis treatments can be carried out without heparin in subsets of patients with high bleeding risks, but even in this patient population, half are forced to switch to heparin bolus dialysis within the first year of treatment ${ }^{2}$. Due to these limitations, other non-thrombogenic, hydrophilic material coatings have been explored, including PHISIO (Sorin) ${ }^{13}$, Trillium (Medtronic) ${ }^{14}$, PMEA polymer ${ }^{15}$, and sulfobetain $^{16}$. Extensive human clinical evaluation of these various alternative 
surface coatings is currently underway; however, no benefit has been shown to date when compared to existing heparin-coated materials ${ }^{17,18}$.

Based on the limited clinical utility of these strategies for reducing thrombosis of extracorporeal circuits, we explored a recently described Slippery Liquid-Infused Porous Surface (SLIPS) approach that was originally inspired by the Nepenthes pitcher plant which employs a surface layer of water to create a low friction surface that prevents attachment of insects ${ }^{19}$. The SLIPS technology creates omniphobic, slippery surfaces by infiltrating porous or roughened substrates with various liquid perfluorocarbons (LPs) that prevent adhesion to the underlying substrate through formation of a stably immobilized, molecularly smooth, liquid overlayer ${ }^{20}$. However, existing medical grade materials, such as polycarbonate (PC), polysulfone (PSU), and polyvinyl chloride (PVC), have highly smooth surfaces. Thus, to create non-adhesive, anti-thrombogenic surfaces that might be useful for clinical medicine in the near-term, we set out to develop a way to modify the SLIPS technology so that it can be applied to these smooth surfaces. This was accomplished by covalently binding a molecular perfluorocarbon layer, or tethered perfluorocarbon (TP), on the material surface and then coating it with a freely mobile layer of LP, such as perfluorodecalin, which has been used extensively in medicine for applications such as liquid ventilation $^{21,22}$, ophthalmic surgery ${ }^{23}$ and as an FDA-approved blood substitute ${ }^{24,}$ 25. Importantly, the TP continues to retain the free LP as a thin mobile liquid layer even when the surface is challenged with a flowing immiscible fluid, such as 
blood (Fig. 1a). We refer to this unique anti-thrombogenic bilayer composed of the TP and LP coating as a Tethered-Liquid Perfluorocarbon (TLP) surface.

\section{RESULTS}

\section{A generic blood repellent surface coating}

To test the anti-adhesive properties of the TLP coating method, we examined surface adhesion of fresh whole human blood on an acrylic surface sloped at an angle of 30 degrees, with or without a TLP coating composed of tethered perfluorohexane and liquid perfluorodecalin. Blood droplets immediately adhered to the control uncoated acrylic surface and left a trail of blood components over the course of $5 \mathrm{sec}$ (Fig. 1b, top, Supplementary Fig. 1 and Supplementary Movie 1). In contrast, when the same surface was coated with TLP, the blood droplet almost immediately slid off the surface $(<0.3 \mathrm{sec})$, and remarkably, there was no evidence of any residual blood trail (Fig. 1b, Supplementary Fig. 1 and Supplementary Movie 2). We quantified blood adhesion to surfaces by measuring the minimum angle required to cause a droplet to slide ("sliding angle") (Fig. 1c). Control uncoated surfaces and surfaces that were modified by coating with either the TP or LP layer alone, all exhibited significant blood adhesion, even when the surface was tilted to 90 degrees (Fig. 1c). In contrast, the minimum angle to cause blood sliding on the fully anti-adhesive TLP acrylic surface was $0.6 \pm 0.2$ degrees. Importantly, this same TLP surface coating was equally effective when applied to multiple other smooth medically relevant plastics, glasses, and metals, including PC, PVC, PSU, polyethylene (PE), polypropylene (PP), polyethylene terephthalate (PET), 
polyimide $(\mathrm{PI})$, polystyrene $(\mathrm{PS})$, borosilicate glass $(\mathrm{G})$, polydimethylsiloxane (PDMS), titanium (Ti), silicon (Si), fluorinated ethylene propylene (FEP), and polytetrafluoroethylene (PTFE) (Figs. 1d and Supplementary Fig. 2). These TLP-coated smooth surfaces also were equally as effective as porous materials (expanded PTFE, colloidal monolayers and nanostructured boehmite) that were infused with LP, the original SLIPS technology (e.SLIPS and B.SLIPS, respectively), which have previously been shown to repel crude oil and ice, as well as anticoagulated animal blood ${ }^{20,26,27}$. Additionally, an acrylic sheet and a commercial PP tube modified with the TLP coating showed no blood adhesion after immersion in human blood, which was in stark contrast to the control (Supplementary Movie 3).

To further demonstrate the novelty of this slippery coating, we tested the ability of TLP-coated acrylic to repel adhesion of a living gecko, which was previously shown to be unaffected by surface coatings on acrylic ${ }^{28}$. Geckos are unique animals in that they are able to hold multiple times their body weight on a vertical surface, and we obtained similar results when geckos were allowed to attach to control acrylic surfaces oriented at up to 90 degrees inclination (Supplementary Movie 4). However, when presented with the TLP-coated acrylic surface, the geckos were unable to hold on, and thus, slipped down before the angle approached vertical (Supplementary Movie 5).

\section{Reduced adhesion and activation of fibrinogen and platelets}

Material-induced thrombosis is mediated via adhesion and activation of two major blood components, fibrinogen and platelets, within minutes after surface 
contact. Cleavage of fibrinogen to fibrin leads to the formation of polymerized fibrin networks that form the backbone of the clot, which becomes intertwined with aggregates of platelets that are simultaneously activated by either interacting directly with the material surface or with surface-bound proteins ${ }^{5}$. We exposed smooth plastic surfaces to fresh, whole human blood containing a low dose of heparin $(0.25 \mathrm{U} / \mathrm{ml})$ because otherwise clotting would initiate immediately. Even with the presence of heparin, control acrylic and polysulfone surfaces formed dense fibrillar networks of polymerized fibrin during the 90-min study (Fig. 2a). While surfaces coated with a TP layer alone (without the LP layer) reduced network formation, adherent fibrils of polymerized fibrin were still detectable. In contrast, coating the acrylic surface with the TLP bilayer fully prevented adhesion of fluorescently labeled fibrin, and TLP-coated polysulfone similarly exhibited only minimal punctate fibrin staining with rare thin fibrils (Fig. 2a). Quantitation of these results confirmed that TLP coating of the acrylic and polysulfone substrates decreased fibrin adhesion and polymerization relative to controls by 6 - and 4 -fold, respectively $(p<0.05)$, and by 3 - and 2 -fold relative to surfaces coated with the TP layer alone (Fig. 2b and Fig. 2c). The reduced adhesion and polymerization of fibrin on the surfaces coated only with the TP layer is important because it suggests that TLP coatings will significantly suppress clot formation, even if the LP layer is displaced after extended exposure to blood. Furthermore, when we measured D-dimer production in whole blood exposed to TLP surfaces as a quantitative measure of fibrin polymerization, levels were significantly lower than those produced on control surfaces (Supplementary Fig. 3). 
To evaluate effects on platelet adhesion, surfaces were analyzed by scanning electron microscopy (SEM) after they were exposed to whole human blood for $30 \mathrm{~min}$. These studies revealed that TLP coatings of acrylic and polysulfone surfaces reduced platelet adhesion by 27 - and 4-fold, respectively, compared to control surfaces ( $p<0.05$, Fig. $\mathbf{2 d}$ and Fig. $2 \mathbf{2 e}$ ). Furthermore, while nearly all platelets adherent to control materials and surfaces coated with TP alone exhibited a flattened or spread morphology consistent with platelet activation, most platelets found on surfaces coated with the full TLP bilayer appeared round, suggesting that they remained in a resting, non-activated state (Fig. $2 f$ and Supplementary Fig. 4). Taken together, these results indicate that TLP surfaces reduce fibrin polymerization and suppress both adhesion and activation of platelets, hence confirming reduced thrombogenicity in vitro.

\section{Reduced thrombosis under flow}

To be medically relevant, the TLP surfaces must remain stable and functional when exposed to physiological shear stresses. When we exposed the surfaces to a constant shear strain rate $\left(1000 \mathrm{~s}^{-1}\right)$ using a rheometer, we found that the LP was displaced from acrylic surfaces that were coated with LP alone with 1 min of exposure to shear, resulting in blood adhesion even when tilted to 90 degrees. In contrast, TLP-coated acrylic surfaces continued to effectively repel blood (sliding angle $<3^{\circ}$ ) even after $16 \mathrm{~h}$ exposure to that same level of fluid shear (Fig. 3a). Also, TLP acrylic surfaces were stable when exposed to a range of shear rates (250-2000 s-1) for 10 min with no change in sliding angle (Supplementary Fig. 5). Thus, these TLP surfaces remain stable under 
hemodynamic fluid shear conditions for extended time periods in vitro because of the higher affinity of LP for the functionalized surface than for blood or water.

We then flowed fresh whole human blood $(0.25 \mathrm{U} / \mathrm{ml}$ heparin at $50 \mathrm{ml} / \mathrm{h})$ through either control or TLP-coated medical grade PVC tubing (1/16" inner diameter). Following rinsing, the remaining adherent proteins were digested off the tubing surface and quantified by a BCA assay (Fig. 3b). These studies revealed that the TLP coating resulted in a 3-fold decrease in protein adhesion compared to control tubing $\left(0.8 \pm 0.2\right.$ versus $2.3 \pm 0.5 \mu \mathrm{g} / \mathrm{cm}^{2}$ respectively) $(p<0.05$; Fig. 3b). Free hemoglobin levels were not significantly different from those measured in baseline blood samples, indicating that the TLP surfaces cause no hemolysis with a single pass of flowing blood (Supplementary Fig. 6).

We then challenged the TLP coating by testing it in a microfluidic device to mimic the physiological microenvironment of blood flowing through a vessel. Fresh whole human blood $(0.5 \mathrm{U} / \mathrm{ml}$ heparin) was infused under a constant pressure with an initial wall shear of $1250 \mathrm{~s}^{-1}$. Blood flowed without occlusion for longer in TLP devices than in LP devices (Fig. 3c), as shown by a significant 2.5fold increase in average clotting time $(p<0.05)$, which corresponds to the time required for the occlusion to reduce flow by half (Supplementary Fig. 7). This indicates that overall thrombosis is significantly reduced on TLP surfaces, as we expected from the reduced fibrin polymerization and suppressed adhesion and activation of platelets we observed in our earlier studies (Fig. 2).

To explore whether this surface coating technology might be useful for extracorporeal circuits in the clinic, the TLP coating was applied to large diameter 
(1/4" ID), medical grade polyvinyl chloride cardioperfusion tubing. No macroscopic or microscopic differences were observed with the application of TP to this tubing (Supplementary Fig. 8a, b). Distinct droplets of blood could also be seen moving along the TLP surface without adhesion, whereas blood adhered to surfaces that were coated with LP alone (Supplementary Fig. 8c). As extracorporeal circuits require peristaltic pumps to drive blood flow, we pumped fresh whole human blood $(0.25 \mathrm{U} / \mathrm{ml}$ heparin) at $3 \mathrm{~L} / \mathrm{h}$ through a closed loop (1/4" ID perfusion tubing) system linked with polycarbonate connectors. These studies showed that there was greatly reduced formation of visually detectable clots within the lumen of the tubing in the TLP-coated PVC loops (Fig. 3d and Fig. 3f). This correlated with a reduction in thrombus weight by $\sim 4$-fold after $2 \mathrm{~h}$ compared to uncoated control loops ( $p<0.05$, Fig. $3 \mathbf{d})$, and the finding that blood platelet counts remained $\sim 3$ fold higher in blood exposed to TLP-coated loops than uncoated loops ( $p<0.05$, Fig. 3e), indicative of reduced platelet adhesion and/or aggregation. In this experiment, platelets traveled through the pump 360 times, which is the equivalent of passing through 150 meters of tubing. This is far harsher than extracorporeal circulation, and hence, the slight platelet loss due to activation from mechanical damage is not to be expected to result in vivo. While the use of peristaltic pumps can sometimes mechanically abrade the tubing where they interface, this did not impair the anti-thrombogenic properties of the TLP surface. Importantly, the TLP coating of the perfusion tubing was consistent along the entire 120 inches of its length (Supplementary Fig. 9a), as verified by similar tilt angles and a high percentage of fluorine atoms throughout 
(Supplementary Table 1). We also found that the TP was stable for 12 months when applied to cannulae and perfusion tubing (Supplementary Fig. 9b), and ethylene oxide sterilization of the TP-coated perfusion tubing did not alter the surface's ability to repel blood (Fig. $\mathbf{3 g}$ ). This coating method therefore provides an effective method to create anti-thrombogenic surfaces on existing medical grade materials which is amenable to rapid commercial translation.

\section{Reduced thrombosis in vivo}

To examine the anti-thrombogenic properties of TLP-modified medical grade materials in vivo, commercially available polyurethane cannulae, polycarbonate connectors and polyvinyl chloride cardiopulmonary perfusion tubing were assembled into an arteriovenous (AV) shunt and modified with the TLP surface coating. We then analyzed its ability to support blood flow for $8 \mathrm{~h}$ in a porcine femoral AV shunt model (Fig. 4a), either with low heparin (30U/kg compared to the $300 \mathrm{U} / \mathrm{kg}$ used for conventional systemic heparinization ${ }^{29}$ ) or without any systemic heparin anticoagulation. In the low heparin group, real-time flow measurements revealed that flow rates remained between 10 and $21 \mathrm{~L} / \mathrm{h}$ in animals implanted with either uncoated control or TLP-modified shunts, although we observed increased flow rate variability in animals with the control (noncoated) materials (Fig. 4b). Importantly, all of the TLP-coated circuits also remained patent for $8 \mathrm{~h}$, maintaining a flow rate of 11 to $18 \mathrm{~L} / \mathrm{h}$, even in the absence of any heparin; in contrast, complete occlusion occurred in 4 out of 5 uncoated circuits in control animals $(p<0.05 ; \mathbf{F i g . ~ 4 c ) . ~ I n ~ t h e ~ n o ~ h e p a r i n ~ g r o u p , ~}$ two animals in the TLP group and one in the control group developed similarly 
low mean arterial pressures (MAP). Nevertheless, despite the increased thrombogenic potential from these decreased flow rates, the circuits remained patent in the TLP animals, whereas the circuit fully occluded in the control animal.

Analysis of circuits from both heparin and no heparin groups after completion of the study revealed that TLP-modified circuits exhibited a marked decrease in occlusive thrombosis in both the arterial and venous sections after 8 h of flow (Fig. 4d). Total thrombus weight in TLP-coated circuits was reduced by 2 -fold compared to controls in the heparin and no heparin groups $(p<0.05$; Fig. 4e). To determine the extent of occlusion throughout the circuits post-explant, lumen areas were measured using computerized image analysis to calculate percent occlusion. In the heparin group, control circuits had $7 \pm 3 \%$ occlusion whereas there was no detectable occlusion in circuits coated with TLP (Fig. 4f and Supplementary Fig. 10). Moreover, even in the absence of heparin, there was a 2.5 -fold reduction in lumen occlusion (from $35 \pm 8 \%$ to $14 \pm 4 \%$ ) in TLPcoated circuits compared to controls $(p<0.05$; Fig. $\mathbf{4 f}$ and Supplementary Fig. 10). These data confirm our in vitro results by showing that the TLP coating significantly reduces the thrombogenicity of medical grade plastics, including widely used PU, PC and PVC, when in direct contact with flowing whole blood for at least $8 \mathrm{~h}$ in vivo. Importantly, in these studies, the TLP coating was as effective at maintaining shunt blood flow with no heparin as control circuits in heparinized animals (Fig. 4b and Fig. 4c). Blood flow was also maintained without producing significant differences in hematologic parameters, pathology or histology (Supplementary Note 1 and Supplementary Table 2), and there was minimal 
leaching of LP into the blood. In the few blood samples where it was detected, free LP levels were at or near the limit of detection $(0.07 \mu \mathrm{g} / \mathrm{ml}$ blood or $\sim 4 \mu \mathrm{g}$ $\mathrm{LP} / \mathrm{kg}$ body weight), which is over 6 orders of magnitude lower than levels of LP that have been used clinically without toxicity $(5.6 \mathrm{~g} \mathrm{LP} / \mathrm{kg} \text { body weight })^{24,30}$.

\section{Biofilm inhibition}

Given the potent ability of the TLP coating to prevent surface adhesion, we also explored whether this surface coating method could prevent adhesion of microorganisms and subsequent biofilm formation on medical materials, which can lead to clinical infections and increased risk of sepsis in patients with indwelling medical devices. In fact, when Pseudomonas aeruginosa bacteria were grown in TLP-coated loops of PVC medical tubing for up to 6.5 weeks, there was an 8-fold reduction in biofilm formation compared to control tubing $(p<0.05)($ Fig. $\mathbf{4 g})$. This ability to suppress biofouling over extended times was confirmed on TLP-modified surfaces, which also significantly reduced $P$. aeruginosa and E. coli bacterial adhesion relative to control surfaces $(p<0.05$; Fig. 4h and Supplementary Fig. 11), similar to results obtained by infusing a roughened surface with a LP using the SLIPS technology ${ }^{31}$. Thus, the TLP coating not only displayed anti-thrombogenicity, it also exhibited potent antibiofouling capabilities. Other anti-biofouling surface modification methods (e.g., sulfobetaine modification) also have been shown to reduce microbial attachment and biofilm formation in vitro, but only for $24 \mathrm{~h}^{16}$.

\section{DISCUSSION}


Taken together, these results demonstrate the TLP coating can be applied to a wide range of different commercially available medical grade materials to create surfaces that are both anti-thrombogenic and anti-biofouling. The TLP surfaces resisted adhesion of fibrin and platelets, suppressed biofouling, and reduced thrombosis when the surfaces came in contact with flowing whole human blood driven by a peristaltic pump in vitro and TLP-coated AV shunts retained their ability to prevent occlusive thrombus formation for at least $8 \mathrm{~h}$ in vivo when implanted in a flowing vascular circuit in pigs.

Sulfobetaine surface coatings also have been shown to reduce thrombus formation when applied to medical grade materials in the presence of slightly reduced heparin concentrations $(0.7$ to $1 \mathrm{U} / \mathrm{mL})$, and clot formation on the outer surface of a sulfobetaine-coated catheter was reduced when it was inserted into a canine jugular vein in the absence of anticoagulants for $4 \mathrm{~h}$, but the surface of the inner lumen was not studied ${ }^{16}$. Sulfobetaine surface coatings also have not been shown to withstand mechanical pumping. In contrast, in our in vivo model, arterial blood flowed through the lumen of the surgically implanted shunt tubing under a much higher pressure and shear rate (10 times higher than the venous model), and thrombosis was reduced for twice as long (8 $\mathrm{h}$ versus 4 ). In addition, in our in vitro studies, the TLP coating prevented coagulation in blood containing $1 / 4$ th the heparin dose used in the sulfobetaine coating study $(0.25 \mathrm{U} / \mathrm{ml}$ vs. $1 \mathrm{U} / \mathrm{ml}$ ), and we did this in blood flow circuits driven by a peristaltic pump (gravity flow was used in the past), which is critical for clinical medical devices that employ extracorporeal circuits. In addition, we successfully created anti- 
thrombogenic surfaces by applying the TLP coating to 20 different medically relevant biomaterials, whereas the sulfobetaine coating method for thrombus reduction has only been demonstrated with polyurethane ${ }^{16}$. Importantly, the TLP process is the first non-heparin coating to show reduced thrombogenicity in vivo on the inner lumen of tubing, which is most clinically relevant for use in extracorporeal circuits.

The reduction in thrombosis we obtained with the TLP-coated circuit is similar to that obtained with other heparin-based coatings in porcine and bovine cardiopulmonary bypass models, but they can leach into blood and require exposure of the blood to extremely high heparin doses $(>50 \mathrm{U} / \mathrm{kg})^{32,33}$. The TLP coating more closely resembles silicone liquid thin films previously used in vitro to delineate blood coagulation mechanisms without anticoagulants for short durations ${ }^{34}$. However, this surface did not prevent platelet adhesion or activation $^{35}$, and it has not been shown to reduce thrombosis in vivo. Hydrophobic surfaces (for example, ePTFE, which is a porous perfluorinated solid material) are clinically approved as vascular grafts ${ }^{36}$; however, these fail to improve the performance of medical devices ${ }^{37}$ because they contain trapped air that can be thrombogenic ${ }^{5,38}$. To our knowledge, there is no other known medical material coating that can effectively suppress occlusive thrombosis in vivo under high pressure and high shear arterial flow in the complete absence of heparin. Thus, as the TLP coating does not leach anticoagulant activity into the blood, it could potentially offer a new way to prevent thrombosis without the complications of heparin anticoagulant therapy. 
The TP we used was covalently coupled to the surface by silane chemistry that is used in dental adhesives, but other reactive groups could be utilized to generate TP layers ${ }^{26}$. The LP perfluorodecalin used here has been previously included in an FDA-approved blood substitute; however, we have found that a variety of LPs, medical grade and non-medical grade, also can repel blood when integrated into a TLP coating (Supplementary Fig. 12). An important caveat for future clinical application of this technology is to avoid use of LPs that evaporate at body temperature, which can induce gaseous emboli formation and significant toxicity $^{39}$. Additionally, it is likely that the surfaces with the tethered perfluorocarbon layer will be sterilized and stored dry, and then the LP will be added to the circuit shortly before, allowing blood to flow through these TLPcoated circuits. This is consistent with the saline priming step that is currently used in clinical studies with extraporeal circuits, and so should be easily integrated into these protocols.

Another advantage of the TLP coating method is that it relies on the use of a low-pressure plasma surface modification procedure commonly used for commercial modification of materials ${ }^{38}$. This can be applied to virtually any material regardless of the complexity of its geometry, without altering the bulk properties of the material ${ }^{40}$. The treatment is also ideal for temperature-sensitive materials, as the plasma occurs at approximately ambient temperature, and for medical devices and implants with complex shapes because the plasma permeates tortuous paths and surface features down to the microscale ${ }^{41}$. This is a significant advantage relative to other surface coatings (e.g. Sulfabetaine) that 
employ a peroxide to activate the surface, which leave some areas untreated and thrombogenic because bubbles are generated ${ }^{42}$.

\section{CONCLUSION}

This TLP coating represents the first surface coating to reduce thrombosis under physiological arterial flow in vivo without the use of soluble anticoagulants, and in tubing that experiences peristaltic pumping ex vivo using significantly reduced levels of anticoagulation ( $0.25 \mathrm{U} / \mathrm{mL}$ heparin). Thus, it can be employed to coat materials used for various types of extracorporeal circuits and it potentially also may be valuable for indwelling devices, including total artificial hearts and ventricular assist devices, as well as needles, Vacutainers ${ }^{\mathrm{Tm}}$, sutures and blood storage bags. Importantly, while there are commercially available surface coating technologies that partially reduce either blood thrombosis or bacterial adhesion, the TLP coating prevents both. Because the TLP coating technology also prevents biofouling, it opens the possibility of creating a new class of medical materials and devices lined by anti-thrombogenic and antibiofouling surfaces that do not require co-administration of antiplatelet, anticoagulant or antibiotic medications when implanted in patients. But the first step is to reduce the need for systemic heparinization and antibiotic drug treatments to prevent related morbidity and fatalities, which would significantly decrease healthcare costs.

Note: Supplementary information is available in the online version of the paper 


\section{ACKNOWLEDGEMENTS}

This work was supported by Defense Advanced Research Projects Agency grant N66001-11-1-4180 and contract HR0011-13-C-0025, and the Wyss Institute for Biologically Inspired Engineering at Harvard University. We thank D. Super, R. Cooper, E. Murray and J. Lee for phlebotomy, T. Ferrante for assistance with fluorescence microscopy, H. Kozakewich for assistance with histology evaluation, and O. Ahanotu for assistance in preparing surfaces. SEM and XPS were conducted at the Center for Nanoscale Systems at Harvard University, a member of the National Nanotechnology Infrastructure Network, which is supported by the National Science Foundation (ECS-0335765). 


\section{ONLINE METHODS}

\section{Modification of Surfaces with Tethered Perfluorocarbon}

Samples were briefly exposed (40 seconds, unless stated otherwise) to low-pressure (150 to $250 \mathrm{mTorr}$ ) radio-frequency (13.56 MHz) oxygen plasma at 100 Watts in order to gently activate the surface to react with the tethered perfluorocarbon silane in a PE-100 plasma system (PlasmaEtch, PE-100, Carson City, NV). Immediately following plasma activation, samples were immersed in a liquid silane solution [5 \% v/v tridecafluoro-1,1,2,2-tetrahydrooctyl trichlorosilane (Gelest, Morrisville PA) in anhydrous ethanol (Sigma, St. Louis MO)] for $1 \mathrm{~h}$ at room temperature. Treated samples were rinsed with anhydrous ethanol (Sigma St. Louis MO), distilled, deionized water (Milli-Q Water Purification System, Millipore), and three times with pure ethanol (Koptec, King of Prussia PA). Rinsed samples were gently blown dry with compressed nitrogen and gently heated in an oven with desiccant at $60^{\circ} \mathrm{C}$ overnight at atmospheric pressure. Medical-grade cardiopulmonary perfusion tubing (Sorin Group, Arvada CO) was exposed to oxygen plasma for $3 \mathrm{~min}$; 8 Fr pediatric arterial cannulae (polyurethane and polycarbonate connectors) (Bio-Medicus, Medtronic, Minneapolis MN), monitoring lines (Smiths Medical, St Paul MN) and 4-way stopcocks (Smiths Medical, St Paul MN) were exposed to oxygen plasma for 2 min. Other materials treated with oxygen plasma for 40 seconds were: poly (methyl methacrylate) (PMMA), polysulfone (PSU), polypropylene (PP), polytetrafluoroethylene (PTFE), polyethylene terephthalate (PET), ultra-high 
molecular weight polyethylene (UHMW PE), polycarbonate (PC) (Goodfellow, Coraopolis PA), polystyrene (PS) (BD Biosciences, Durham NC), polyvinyl chloride (PVC), fluorinated ethylene propylene (FEP), perfluoroalkoxy (PFA), titanium, polyimide (PI), stainless steel (McMaster-Carr, Robbinsville NJ), glass cover slip (VWR, Radnor PA), polydimethylsiloxane (PDMS) (Dow Corning, Midland MI), Aeos ${ }^{\mathrm{TM}}$ HyFlex expanded polytetrafluoroethylene (ePTFE) (Zeus, Orangeburg SC), silicon wafer (Ted Pella, Redding CA). Atomic force microscopy revealed minimal change in surface roughness after TP coating $(3.4 \pm 1 \mathrm{~nm})$ compared to control acrylic $(2.0 \pm 0.2 \mathrm{~nm})($ mean $\pm S D, n=3)$.

\section{Sliding Angle of Surfaces}

The angle at which a droplet of liquid begins to move across a surface (sliding angle) was measured for the samples using a manual goniometer (Thor Labs GN05/M). Samples were dip-coated in perfluorodecalin (PFD) (FluoroMed, APF-140HP (sterile, high purity), Round Rock TX) immediately before measurement and the sample was placed on top of the leveled goniometer. The amount of PFD on the surface was $4-6 \mu \mathrm{l} / \mathrm{cm}^{2}$ as measured by an analytical balance after dip coating. For tilting at 30 degrees, $200 \mu \mathrm{l}$ of citrated whole human blood (see method Human blood samples) was placed gently onto surfaces in $\sim 50 \mu \mathrm{l}$ droplets. For quantification of sliding angle, a $5-\mu \mathrm{L}$ droplet of citrated whole human blood was gently placed on the surface. The sample was then tilted until the droplet was observed sliding along the surface. For samples that did not slide by 15 degrees, a custom built setup smoothly tilted the sample to 90 degrees. Samples still adherent at 90 degrees were noted as a sliding 
angle of 90 degrees. Sliding angle measurements were obtained on TLP acrylic with alternate LPs: perfluorohexane (PFH, Sigma), perfluorooctane (PFO, Sigma), 1-bromoperfluorooctane (PFOB, Oakwood Products), perfluoroperhydrophenanthrene (Vitreon, FluoroMed APF-215HP (sterile, high purity)), perfluorotripentylamine (FC-70, HamptonResearch), perfluorotributylamine/perfluorodibutylmethylamine (FC-40, Santa Cruz Biotechnology), 3-ethoxy-1,1,1,2,3,4,4,5,5,6,6,6-dodecafluoro-2-trifluoromethylhexane (HFE-7500, 3M), perfluoropolyether oils (Krytox 101, Krytox 103, food grade Krytox FG-40, Dupont)).

\section{Elemental Analysis Methods}

X-ray photoelectron spectroscopy (XPS) was performed on a Thermo Scientific K-Alpha X-Ray Photoelectron Spectrometer (Thermo Scientific). Samples were prepared with TP treatment as described above. PVC tubing was stored under vacuum prior to XPS analysis to accelerate outgassing of plasticizers. Auto-Analysis scans (XPS displacement tolerance of $8 \mathrm{eV}$, Auger displacement tolerance of $12 \mathrm{eV}$ ) were performed and analyzed using the Thermo Scientific Avantage Data System v5.915 (Thermo Scientific). Three TLP samples and 1 control sample were tested. Three points were selected on each sample with a spot size of $30 \mu \mathrm{m}$. Five survey scans (binding energy range from -4 to $1350 \mathrm{eV}$ ) were averaged at each point to identify potential elements on the sample surface. Based on the potential elements identified during the survey scans, higher resolution individual elemental analysis scans were then performed for each elemental range and averaged over 10 scans. XPS confirmed the highly 
fluorinated surface layer (TP) on acrylic, PC, PSU and PVC tubing

\section{(Supplementary Table 1).}

\section{Gecko adhesion}

Study approval was obtained from Boston Children's Hospital Institutional Animal Care and Use Committee (protocol number 13-10-2552). One eyelashcrested (Rhacodactylus ciliatus) gecko was placed inside an acrylic cylinder and tilted slowly from horizontal to vertical. This was repeated with the gecko inside a TLP treated acrylic cylinder and the tilting was stopped when the gecko slid to the bottom of the cylinder. This was repeated a total of 3 times with the gecko allowed to recuperate for 1 week between experiments. Videos are representative of the three trials.

\section{Surfaces under Shear}

Surfaces were exposed to shear using a rheometer (TA Instruments, Model AR-G2) with a 40-mm diameter, $2^{\circ}$ angle cone-and-plate setup (TA Instruments, Model 513406.905) and Peltier plate (TA Instruments, Model 531051.901). Acrylic sheets were cut into 40-mm diameter discs using a laser cutter (Epilog Legend 36EXT). These discs were then aligned with the cone platen, and adhered to the bottom Peltier plate platen with adhesive (3M, St Paul MN). The samples were then lubricated with just enough PFD to cover the surface of the acrylic disc (approximately 500-600 $\mu \mathrm{L}$ of PFD). Approximately 2 $\mathrm{mL}$ of $35 \% \mathrm{v} / \mathrm{v}$ glycerol (Sigma, St. Louis MO) in water was applied on top of the lubricated acrylic disc. The cone platen was then lowered $50 \mu \mathrm{m}$ above the acrylic disc, and the excess PFD and glycerol was then pushed out from between 
the platens. This excess fluid was removed to allow a meniscus of glycerol solution to form between the two platens. Solvent rings were then placed around the setup to minimize evaporation of the liquids. For studies with shear rates below $500 \mathrm{~s}^{-1}$, a 10-min conditioning step was performed at $500 \mathrm{~s}^{-1}$ to ensure the excess PFD was removed.

\section{Scanning electron microscopy}

Samples that had been in contact with blood or bacteria were fixed with $2.5 \%$ glutaraldehyde in $0.1 \mathrm{M}$ sodium cacodylate buffer (Electron Microscopy Sciences, Hatfield PA) for $1 \mathrm{~h}, 1 \%$ osmium tetroxide in $0.1 \mathrm{M}$ sodium cacodylate (Electron Microscopy Sciences, Hatfield PA) for $1 \mathrm{~h}$, dehydrated in ascending grades of ethanol, and chemically dried with hexamethydisilazane (Electron Microscopy Sciences, Hatfield PA) in a desiccator overnight before being mounted and sputter coated with a thin layer of gold/palladium and imaged on a Zeiss Supra55VP microscope.

\section{Human blood samples}

Approval for studies involving human subjects was obtained from Harvard University Faculty of Medicine Committee on Human Studies (protocol number M20403-101). Whole human blood was obtained from healthy volunteers with informed consent who were non-smokers and had not taken aspirin 2 weeks prior to donation. Blood was drawn by standard venipuncture into no additive Vacutainers (Becton Dickenson, Franklin Lakes NJ). A discard tube was drawn first, then heparin (1000 U/ml) (APP Pharmaceuticals, Schaumburg IL) was 
added to subsequent tubes to a final concentration dependent on the assay.

Assays were based on ISO-10993-4 for evaluation of medical devices ${ }^{43}$.

\section{Whole blood adhesion assay}

Wells of a 24 -well plate were blocked with $1 \%(\mathrm{w} / \mathrm{v})$ bovine serum

albumin (BSA) (Sigma A3803, St. Louis MO) in saline for 30 min and rinsed with saline. Samples $(11 \times 8 \mathrm{~mm})$ of $100-\mu \mathrm{m}$ thick polysulfone or $1 / 16$ inch thick acrylic were control, TP or dip coated in PFD to generate TLP. Samples were incubated in blocked wells with heparinized whole human blood $(0.25 \mathrm{U} / \mathrm{ml}$ to prevent immediate clotting whilst retaining the ability of blood components to be activated by surfaces). Sample order was randomized for incubation with blood. Fluorescently labeled fibrinogen $(150 \mu \mathrm{g} / \mathrm{ml}$, Invitrogen, Carlsbad CA, $90 \%$ clottable fraction) was added to the blood which was then incubated with samples for increasing time points on an orbital shaker. D-dimer concentration was measured in blood from one donor by ELISA after 60 min (Sekisui Diagnostics, LLC, Stamford, CT) according to the manufacturers instructions. Acrylic and polysulfone samples were washed 3 times in normal saline $(0.9 \%$ sodium chloride; Baxter Healthcare, Cambridge MA) and fixed for $1 \mathrm{~h}$ with $2.5 \%$ glutaraldehyde in $0.1 \mathrm{M}$ sodium cacodylate buffer before imaging with a Hamamatsu 9100-02 EMCCD camera on a Zeiss Axio Observer Z1 fluorescent microscope using a $20 \mathrm{X}$ objective and Metamorph software. Quantification was carried out using ImageJ (http://imagej.nih.gov/ii/) on 8 unadjusted images from each sample. The images from each experiment were converted to binary images and generated into a stack. The threshold was manually set to 6 for all 
images, and the area covered by fibrin was extracted from the measurements. A maximum fibrin clot area of $60-70 \%$ was quantified due to the fibrillar nature of the thrombus on the surface and imaging on the surface focal plane. Each experiment was done with 2 replicates per donor and repeated with 3 donors. Representative images in Fig. 2a were converted to green and brightness and contrast was uniformly adjusted to all for clarity. Samples from the 30 min time point were processed for SEM as described above. Scanning electron micrographs from 4 areas per sample were obtained, and a blinded researcher counted the number of adhered platelets. Non-activated platelets were differentiated from activated platelets by their round or dendritic morphology, as defined by Goodman ${ }^{44}$. Non-activated platelets were subsequently counted, identified by rounded morphology with zero flattened protrusions. The number of non-activated platelets was divided by total adherent platelets per field of view, and multiplied by 100 percent to obtain the percent activated platelet value.

\section{Blood Flow in Small-Diameter Medical Grade Tubing}

TP monitoring lines (1/16" ID by 48" length) were primed with $3 \mathrm{~mL}$ of PFD. Control and TLP tubing were subsequently primed with saline. Human heparinized whole blood $(0.25 \mathrm{U} / \mathrm{mL})$ at room temperature was drawn through the monitoring line at a flow rate of $60 \mathrm{~mL} / \mathrm{hr}$ by a syringe pump (Harvard Apparatus, Holliston MA) by withdrawal. This flow rate corresponds to a shear strain rate of $40 \mathrm{~s}^{-1}$ and was maintained over $20 \mathrm{~min}$. Blood was collected into a 20-mL syringe (Becton Dickinson, Franklin Lakes NJ) containing a 1:9 volume of $3.8 \%$ Sodium Citrate (Ricca Chemical Company, Arlington TX). Tubing was 
rinsed with $20 \mathrm{~mL}$ normal saline at a flow rate of $60 \mathrm{~mL} / \mathrm{hr}$. Blood samples were collected into EDTA vacutainers (Becton Dickinson, Franklin Lakes NJ) for 18parameter complete blood count using the VetScan HM2 Hematology System (Abaxis, Union City, CA). Tubing was incubated with buffer composed of 0.04 $\mathrm{U} / \mathrm{ml}$ plasmin (Hematologic Technologies Inc., Essex Junction VT), normal saline, $6.25 \mathrm{mM} \mathrm{CaCl}_{2}$, and $4.68 \mathrm{mM} \mathrm{MgCl} 2$ for $1 \mathrm{~h}$ at $37^{\circ} \mathrm{C}$. Samples were then centrifuged at $200 \mathrm{~g}$ for $10 \mathrm{~min}$ to sediment red blood cells, and the total protein concentration was measured in the supernatant by a BCA protein assay following manufacturer's protocol (Thermo Fisher Scientific, Waltham, MA). The mass of protein was then divided by the area of the inner lumen of the tubing $\left(60.8 \mathrm{~cm}^{2}\right)$ to determine the protein adsorbed $/ \mathrm{cm}^{2}$. Further, we measured adsorption of physiological levels of bovine serum albumin $(50 \mathrm{mg} / \mathrm{mL})$ to test the hypothesis that this assay reflects the adhesion of abundant plasma proteins that are less relevant to thrombus formation. We detected very small amounts of albumin bound to the surface $\left(0.035 \pm 0.002 \mu \mathrm{g} / \mathrm{cm}^{2}\right)$ that were barely above background levels. Each paired (TLP vs. control) experiment was done with 3 donors.

\section{Blood Flow in Microfluidic Channels}

TP treated and control microfluidic PDMS microdevices with microchannels $200 \mu \mathrm{m}$ wide by $75 \mu \mathrm{m}$ tall were primed with LP. Human heparinized whole blood $(0.5 \mathrm{U} / \mathrm{ml})$ was flowed in the constant pressure mode with a calculated initial wall shear rate of $1250 \mathrm{sec}^{-1}\left(50 \mathrm{dynes} / \mathrm{cm}^{2}\right)$ via syringe pump (PHD Ultra CP, Harvard Apparatus) with disposable pressure sensor (PendoTECH). Clotting times were derived by finding the time for flow to reduce 
to half its initial value, from a sigmoidal decay fitted curve ( $n=3$ donors). Because the microfluidic device required more extensive time for setup, we used a slightly higher heparin dose to prevent coagulation prior to TLP exposure before the analysis was carried out. This dose $(0.25 \mathrm{U} / \mathrm{mL})$ was still very low in comparison to that commonly used in standard in vitro coagulation assays (5 to $15 \mathrm{U} / \mathrm{mL}$ ).

\section{Blood Flow in Large Diameter Medical-Grade Tubing}

TLP PVC tubing (1/4" ID by 15" length) was primed with $10 \mathrm{~mL}$ of PFD. Control and TLP tubing were subsequently washed with saline. PVC tubing was connected with control or TLP 1/4" ID polycarbonate barbed connectors and filled with human heparinized whole blood $(0.25 \mathrm{U} / \mathrm{mL})$. Blood was pumped at a flow rate of $3 \mathrm{~L} / \mathrm{hr}$ (shear rate of $250 \mathrm{~s}^{-1}$ ) by a peristaltic pump (Cole Parmer, MasterFlex L/S, Vernon Hills, IL) for $2 \mathrm{~h}$. Blood was filtered through a preweighed $40 \mu \mathrm{m}$ cell strainer and air-dried before thrombus weight was obtained. Blood was collected into EDTA Vacutainers for CBC analysis at the time of venipuncture, and again after being pumped through the tubing and filtered through the cell strainer. Reported values of of the percentage of platelets remaining in blood were calculated as the platelets in the sample collected after pumping divided by the platelets in the sample collected before tubing multiplied by 100 percent. Each experiment was done with 3 donors.

\section{Porcine arteriovenous shunt model}

Study approval was obtained from Boston Children's Hospital Institutional Animal Care and Use Committee (protocol number 12-06-2202) and conducted in an AAALAC accredited USDA registered facility. A total of 15 female Yorkshire 
swine weighing $24-35 \mathrm{~kg}$ were used in this study. Animals were randomly assigned to control or TLP groups (no blinding was performed as this was not possible due to the application of LP immediately prior to implantation of the shunt). No power analysis was performed to determine sample size. One group of animals received $30 \mathrm{U} / \mathrm{kg}$ heparin at the time of circuit placement $(n=3$ control and $n=2$ TLP). One animal in the TLP group was excluded as the TLP treatment was performed outside the pre-specified parameters specified above; the pressure gauge on the plasma system was found to be out of calibration. The second group received no heparin $(n=5$ control and $n=4$ TLP). The control group received unmodified extracorporeal materials and the TLP group received an extracorporeal circuit in which all the materials had been treated with TP, sterilized with ethylene oxide and lubricated with $10 \mathrm{ml}$ sterile LP (as received from Fluoromed) and drained within 10 min prior to implantation. The amount of PFD on the circuits at implantation was 0.8-1.2 $\mathrm{g}$.

Animals were anesthetized with intramuscular injections of atropine $(0.04$ $\mathrm{mg} / \mathrm{kg})$, telazol $(4.4 \mathrm{mg} / \mathrm{kg})$ and xyalzine $(2.2 \mathrm{mg} / \mathrm{kg})$ and maintained on isoflurane $(1.5-2.5 \%)$ and oxygen $(1.2 \mathrm{~L} / \mathrm{min})$ delivered through a $7 \mathrm{~mm}$ endotracheal tube using a positive pressure ventilator. The animal was placed in the supine position and a 6 Fr percutaneous sheath catheter was placed in the left femoral artery for pressure monitoring. A $20 \mathrm{~g}$ intravenous cannula was placed in the left marginal ear vein for administration of drugs and a 10 Fr Foley catheter was placed for urinary drainage. The AV shunt was established via cutdown of the right femoral artery and vein and insertion of 8 Fr pediatric arterial 
cannulae (Bio-Medicus, Medtronic, Minneapolis MN). The cannulae were connected to the extracorporeal circuit, which consisted of 25 inches of 1/4" inch ID perfusion tubing (Sorin Group) with a 1/4" barbed connector (Sorin Group) and large bore 4-way stopcock (with rotating male luer lock, Baxter) placed 3 inches from the venous cannula. The perfusion tubing was filled with $1 \mathrm{U} / \mathrm{ml}$ heparinized saline during placement, which was fully drained prior to establishing circuit flow. Shunt implantation time was greater than 2 times the activated clotting time. The heart rate, pulse rate, arterial pressure, oxygen saturation, $\mathrm{CO}_{2}$ level, temperature and respiratory rate were monitored throughout the experiment. Vitals were maintained at physiological levels and body temperature was maintained at $37^{\circ} \mathrm{C}$ via a heat mat. After $8 \mathrm{~h}$, animals were given $300 \mathrm{U} / \mathrm{kg}$ heparin and euthanized with an intravascular lethal dose of Fatal Plus.

\section{In vivo blood sampling and flow measurements}

At baseline (prior to $\mathrm{AV}$ cannulation), time $0 \mathrm{~h}$ (immediately after arteriovenous circuit flow was established), time $3.5 \mathrm{~h}$, and time $7.5 \mathrm{~h}$, blood samples $(20 \mathrm{ml})$ were taken for complete blood count (CBC), blood gas and chemistry profiles, and clotting times (PTT, APTT, ACT) (EDTA, heparin and citrate Vacutainers, respectively; Becton Dickenson, Franklin Lakes NJ). Flow was measured in the mid point of the perfusion tubing using a clamp-on tubing flow sensor connected to a TS410 flow meter module (Transonic, Ithaca NY) for 15 min after circuit flow was established and every 30 min thereafter for $\sim 15$ min each time. Occlusion was measured post-explant by taking photographs of cross 
sections of the cannulae and tubing and digitally determining the area of the lumen and area of the thrombus to calculate the percent occlusion.

\section{Histology}

Organ samples (lung, liver, kidney, spleen and brain) were fixed in $10 \%$ neutral buffered formalin (Electron Microscopy Sciences, Hatfield PA) for $24 \mathrm{~h}$ at room temperature. Tissue was processed and stained with hematoxylin and eosin (H\&E) at Boston Children's Hospital Histopathology services. No evidence of thrombi or microemboli was found in either control or TLP lung sections.

\section{Gas Chromatography/Mass Spectrometry (GC/MS)}

Blood samples $(2 \mathrm{ml})$ were extracted with methyl nonafluoroisobutylether (HFE) (Miller-Stephenson) and analyzed by gas chromatography/mass spectrometry ${ }^{45}$. The limit of detection was determined by multiplying the standard deviation of the baseline response by 3 ; this was converted into a clinical dose by multiplying the LOD by $60 \mathrm{ml}$ of blood $/ \mathrm{kg}$ of body weight.

\section{Biofouling Assay}

Control and TP samples of acrylic $\left(11 \times 8 \mathrm{~mm}, 1 / 16^{\text {th }}\right.$ inch thick $)$ were sterilized with ethanol (pure ethanol, 200 proof) and allowed to air dry in a biological laminar flow hood for 30 min. Samples were transferred to 24 well plate wells and sterile LP was added to TP samples. LP was removed and all samples were immediately incubated with $10^{5}$ CFU of E. coli (ATCC 8739) in RPMI (Invitrogen, Carlsbad CA) for $48 \mathrm{~h}$ at $37^{\circ} \mathrm{C}$. After culture, samples were washed 3 times in PBS and assayed for biofilm formation using crystal violet (Becton Dickenson, Sparks MD). Samples were incubated with $0.1 \%$ crystal violet for $1 \mathrm{~h}$ 
and washed 6 times with distilled water. The crystal violet was solubilized with $10 \%$ acetic acid for $10 \mathrm{~min}$ before $100 \mu \mathrm{l}$ was transferred to a 96 well plate and the absorbance measured using a Bioplex plate reader at $590 \mathrm{~nm}$. The inoculum and cultures of the control and TLP samples after $48 \mathrm{~h}$ were confirmed to be viable by plating.

PE and PET samples (1x1 inch) were incubated with $P$. aeruginosa (a clinical isolate from Brigham and Women's Specimen Bank (protocol number M20403-101)) overnight. Samples were washed in PBS, stained with crystal violet for $15 \mathrm{~min}$, washed with distilled water three times and photographed or fixed for SEM. Bacterial adhesion under continuous flow was tested using a modified Chandler loop setup. The $P$. aeruginosa bacteria were cultured in RPMI Media (Life Technologies, Carlsbad CA) at $37^{\circ} \mathrm{C}$. Control and TLP loops ( $3 \mathrm{~mm}$ inner diameter) were filled with $P$. aeruginosa cultures $\left(10^{5} \mathrm{CFU} / \mathrm{ml}\right)$ and incubated at $25^{\circ} \mathrm{C} .1 .5$ weeks and 6.5 weeks after initial inoculation, two $1 \mathrm{~cm}$ segments of tubing were assayed for biofilm formation. To measure bacterial adhesion on the tubing surface we utilized a novel FcMBL fusion protein ${ }^{46}$. Briefly, the carbohydrate recognition domain of Mannose Binding Lectin (MBL) was fused to the Fc domain of human $\lg G$ and recombinantly expressed in chinese hamster ovary cell lines. FcMBL was conjugated with horseradish peroxidase (FcMBL-HRP) using the Lightning Link-HRP Antibody Labeling Kit (Novus Biologicals, Littleton CO) and used as a detection antibody for ELISA-based detection of $P$. aeruginosa on tubing segments. Tubing was washed in Trisbuffered saline, $0.1 \%$ Tween 20 (TBS-T) (BostonBioProducts, Ashland MA) 
supplemented with $5 \mathrm{mM} \mathrm{CaCl}_{2}$ (BostonBioProducts, Ashland MA), followed by incubation with FcMBL-HRP in $3 \%$ BSA in TBS-T $5 \mathrm{mM} \mathrm{CaCl}_{2}$. The colorimetric reaction was performed with the Pierce 1-Step TMB substrate (Thermo Fisher Scientific, Waltham MA) according to the manufacturer's protocol, and absorbance at $450 \mathrm{~nm}$ was measured. Bacterial titres were established by comparison to a standard curve of $P$. aeruginosa. The culture removed from the loops after 6.5 weeks was confirmed to be viable by plating.

\section{Statistical analysis}

Data are expressed as mean $\pm S D$ for sliding angles, and mean \pm SEM for all other data. In vitro assay sample size was predetermined with 3 separate donors to account for biological variability. Data were statistically analyzed by paired, two-tailed student's t test (Fig. 3b), unpaired, two-tailed student's t test (Fig. 3d-e, Supplementary Fig. 6), and two-way analysis of variance (ANOVA) with Bonferroni post-hoc analysis for multiple comparison (Fig. 2b-e, Supplementary Fig. 3, Fig. 4e-g). A p value of $<0.05$ was considered significant (as indicated in the figures by an asterisk). Graphpad Prism version 6.00 (Graphpad Software, San Diego, CA) was used for statistical analysis. 


\section{FIGURE CAPTIONS}

Figure 1. TLP-coated surfaces repel whole human blood. (a) Schematic of blood repellency on tethered liquid perfluorocarbon (TLP) surfaces showing the tethered perfluorocarbon (TP) bound to a substrate through plasma activation and silane treatment, which then allows a stable film of liquid perfluorocarbon (LP) to adhere to the surface. (b) Surfaces without the tethered or liquid perfluorocarbon (-TP/-LP) show adhesion of a blood droplet (50 $\mathrm{\mu l}, 3.2 \%$ sodium citrate) on the $30^{\circ}$ angled surface, low velocity and residence over 5 seconds (Control, upper panels). When both the tethered and liquid perfluorocarbons are

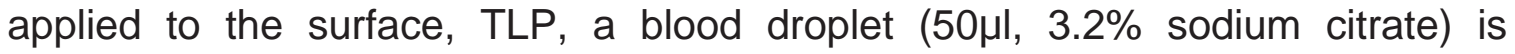
immediately repelled and slides down the surface at an incline of $30^{\circ}$ within 0.3 seconds (lower panels). (c) Graph showing the minimum angle that allowed whole blood ( $5 \mu$ l droplet, $3.2 \%$ sodium citrate) to slide on the different surface treatments $(n=3)$. (d) Graph showing that TLP can be applied to a wide range of materials with a low whole blood sliding angle (black bars) compared to control surfaces (grey bars) comprising polycarbonate (PC), polyvinyl chloride (PVC), polysulfone (PSU), polyethylene (PE), polypropylene (PP), polyethylene terephthalate (PET), polyimide (PI), polystyrene (PS), borosilicate glass $(\mathrm{G})$, titanium (T), silicon wafer (Si), fluorinated ethylene propylene copolymer (FEP), polytetrafluoroethylene (PTFE), expanded polytetrafluoroethylene (ePTFE) Slippery Liquid Infused Porous Surface (SLIPS) (e.SLIPS) and boehmite SLIPS (B.SLIPS) $(n=3)$. 
Figure 2. Whole blood interactions with TLP surfaces. (a) Fluorescent micrographs of acrylic (upper panels) or polysulfone (lower panels) pieces $(11 \times 8 \mathrm{~mm})$ after $90-\mathrm{min}$ incubation with fresh human blood containing heparin $(0.25 \mathrm{U} / \mathrm{ml})$ and fluorescent fibrinogen $(150 \mu \mathrm{g} / \mathrm{ml})$ showing polymerized fibrin networks on the control (left), decreased network formation on TP (middle) and punctate staining with minimal network formation on TLP (right). Scale bar, $50 \mu \mathrm{m}$. (b) Graph showing a reduction of percent fibrin covered area on TLP (full line) acrylic compared to control (dotted line) and TP (dashed line). Percentages quantified using ImageJ ( ${ }^{*} p<0.05$ compared to control, SEM). (c) Graph showing a reduction on TLP (full line) polysulfone compared to control (dotted line) and TP (dashed line) $\left({ }^{*} p<0.05\right.$ compared to control, SEM). (d) Graph showing a 27fold reduction in platelet adhesion (number per $150 \mu \mathrm{m}^{2}$ ) on TLP acrylic compared to control and (e) $\sim 4$-fold reduction on TLP polysulfone $\left({ }^{*} p<0.05\right.$, SEM). (f) Scanning electron micrographs of acrylic surfaces after 30-min incubation shows reduced platelet adhesion on TLP compared to control and TP. Scale bar $10 \mu \mathrm{m}$. Insets show platelet morphology, white arrows show platelets. Inset scale bar $2 \mu \mathrm{m}$. All data are from experiments with 3 separate donors.

Figure 3. Stability and thrombogenicity of TLP surfaces. (a) Graph showing the low blood sliding angle on TLP acrylic discs (black bars) remains stable over 1000 min under a constant shear rate of $1000 \mathrm{~s}^{-1}$ while LP acrylic discs (grey bar) failed and had a 90 degree sliding angle after 1 min. (b) Decreased protein adhesion on TLP PVC, measured by BCA assay, after whole human blood flow $(0.25 \mathrm{U} / \mathrm{ml}$ heparin) through 1/16" ID PVC tubing for $20 \mathrm{~min}$ at 
$50 \mathrm{ml} / \mathrm{h}$. (c) Graph showing maintenance of whole human blood flow $(0.5 \mathrm{U} / \mathrm{ml}$ heparin at $1250 \mathrm{~s}^{-1}$ ) in TLP microfluidic channels (PDMS) over 50 min (black line) while LP channels occluded after $\sim 15$ min (grey line). (d) Graph showing reduced thrombus weight in TLP medical grade PVC (black bar) compared to control (grey bar, $\left.{ }^{*} p<0.05\right)$ after blood was pumped in a closed loop at $3 \mathrm{~L} / \mathrm{h}$ for $2 \mathrm{~h}$. (e) Graph showing an increased percentage of platelets remained in the blood after exposure to TLP tubing (black bar) compared to control tubing (grey bar, $\left.{ }^{*} p<0.05\right)$ after the blood was pumped in a closed loop at $3 \mathrm{~L} / \mathrm{h}$ for $2 \mathrm{~h}$. (f) Photographs of filtered thrombi after blood was pumped in a closed loop at $3 \mathrm{~L} / \mathrm{h}$ for $2 \mathrm{~h}$ in control (upper image) and TLP medical grade PVC (lower image). Scale bar 5mm. (g) Photographs of control (left) or TLP (right) cardioperfusion tubing after sterilization with ethylene oxide (EtO) after it had been exposed to porcine blood for $2 \mathrm{~min}$. Scale bar $5 \mathrm{~mm}$. All data are from experiments with 3 separate donors.

Figure 4. Thrombogenicity of TLP-coated circuits in a porcine arteriovenous shunt model. (a) Schematic of the porcine arteriovenous shunt model showing placement of the cannulae in the femoral artery and vein connected by tubing. Black arrows indicate direction of blood flow driven by the heart. (b) Real-time measurements of flow rate (10 second average) at the midpoint of the circuit showing that in heparinized animals (+ Heparin), flow rate is maintained for $8 \mathrm{~h}$ in both control (grey line) and TLP (black line). (c) Real-time flow rate measurements in non-heparinized animals (- Heparin) revealed 4 out of 5 control circuits (grey line) occlude, whereas TLP circuits (black line) remain 
patent for $8 \mathrm{~h}\left({ }^{*} p<0.05\right)$. (d) Photographs of polyurethane (PU) cannulae, polycarbonate connectors (PC) and polyvinyl chloride (PVC) tubing of TLP (top) and control (bottom) circuits after $8 \mathrm{~h}$ of blood flow. Arrows indicate direction of blood flow through arterial (Art) or venous (Ven) cannula. Increased thrombus is visible in the control circuit. Scale bar $5 \mathrm{~mm}$. (e) Thrombus weight in the TLP circuits (black bars) is reduced under both + Heparin and - Heparin conditions compared to control circuits (grey bars) $\left({ }^{*} p<0.05\right.$, SEM). (f) Percent occlusion of circuits showing that TLP (black bar) has minimal occlusion compared to control (grey bar) in the presence or absence of heparin $\left({ }^{*} p<0.05\right.$, SEM). (g) Graph showing biofilm formation on control PVC (grey bar) and TLP PVC (black bar) for $1.5\left({ }^{*} p<0.05\right.$, SEM) and 6.5 weeks. (h) Photographs of crystal violet stained $P$. aeruginosa on polyethylene control (left) and TLP (right) surfaces after culturing for $16 \mathrm{~h}$. Scale bar $5 \mathrm{~mm}$. Insets show SEM images. Scale bar $2 \mu \mathrm{m}$. 


\section{REFERENCES}

1. McCarthy, P.M. \& Smith, W.A. Mechanical Circulatory Support--a Long and Winding Road. Science 295, 998-999 (2002).

2. Shen, J.I., Mitani, A.A., Chang, T.I. \& Winkelmayer, W.C. Use and safety of heparin-free maintenance hemodialysis in the USA. Nephrol. Dial. Transplantation 28, 1589-1602 (2013).

3. Segesser, L.K.v. et al. Risk and benefit of low systemic heparinization during open heart operations. Ann. Thorac. Surg. 58, 391-398 (1994).

4. Conn, G. et al. Is there an alternative to systemic anticoagulation, as related to interventional biomedical devices? Expert Rev. Med. Devices 3, 245-261 (2006).

5. Werner, C., Maitz, M.F. \& Sperling, C. Current strategies towards hemocompatible coatings. J. Mater. Chem. 17, 3376-3384 (2007).

6. Cronin, R.E. \& Reilly, R.F. Unfractionated Heparin for Hemodialysis: Still the Best Option. Semin. Dial. 23, 510-515 (2010).

7. Finkel, K.W. \& Podoll, A.S. Complications of Continuous Renal Replacement Therapy. Semin. Dial. 22, 155-159 (2009).

8. Shepherd, G., Mohorn, P., Yacoub, K. \& May, D.W. Adverse Drug Reaction Deaths Reported in United States Vital Statistics, 1999-2006. Ann. Pharmacother. 46, 169-175 (2012).

9. Peppas, N. \& Langer, R. New challenges in biomaterials. Science 263, 1715-1720 (1994). 
10. Larm, O., Larsson, R. \& Olsson, P. A new non-thrombogenic surface prepareed by selective covalent bidning of heparin via a modified reducing terminal residue. Biomater. Med. Devices Artif. Organs 11, 161 - 173 (1983).

11. Bannan, S. et al. Low heparinization with heparin-bonded bypass circuits: Is it a safe strategy? Ann. Thorac. Surg. 63, 663-668 (1997).

12. Lobato, R.L. et al. Anticoagulation management during cardiopulmonary bypass: A survey of 54 North American institutions. J. Thorac. Cardiovasc. Surg. 139, 1665-1666 (2010).

13. Thiara, A.S. et al. Comparable biocompatibility of Phisio- and Biolinecoated cardiopulmonary bypass circuits indicated by the inflammatory response. Perfusion 25, 9-16 (2010).

14. Palanzo, D.A. et al. Effect of Carmeda® BioActive Surface coating versus Trillium $^{\mathrm{TM}}$ Biopassive Surface coating of the oxygenator on circulating platelet count drop during cardiopulmonary bypass. Perfusion 16, 279-283 (2001).

15. Suhara, $\mathrm{H}$. et al. Efficacy of a new coating material, PMEA, for cardiopulmonary bypass circuits in a porcine model. Ann. Thorac. Surg. 71, 1603-1608 (2001).

16. Smith, R.S. et al. Vascular Catheters with a Nonleaching PolySulfobetaine Surface Modification Reduce Thrombus Formation and Microbial Attachment. Sci. Transl. Med. 4, 153ra132 (2012). 
17. Kutay, V. et al. Biocompatibility of Heparin-Coated Cardiopulmonary Bypass Circuits in Coronary Patients With Left Ventricular Dysfunction Is Superior to PMEA-Coated Circuits. J. Card. Surg. 21, 572-577 (2006).

18. Reser, D. et al. Retrospective analysis of outcome data with regards to the use of Phisio ${ }^{\circledR}$-, Bioline ${ }^{\circledR}$ - or Softline $\AA$-coated cardiopulmonary bypass circuits in cardiac surgery. Perfusion 27, 530-534 (2012).

19. Bohn, H.F. \& Federle, W. Insect aquaplaning: Nepenthes pitcher plants capture prey with the peristome, a fully wettable water-lubricated anisotropic surface. Proc. Natl. Acad. Sci. U S A 101, 14138-14143 (2004).

20. Wong, T.-S. et al. Bioinspired self-repairing slippery surfaces with pressure-stable omniphobicity. Nature 477, 443 - 447 (2011).

21. Clark Jr., L. \& Gollan, F. Survival of Mammals Breathing Organic Liquids Equilibrated with Oxygen at Atmospheric Pressure. Science 152, 1755 1756 (1966).

22. Clark Jr., L.C. et al. Perfluorocarbons Having a Short Dwell Time in the Liver. Science 181, 680 - 682 (1973).

23. U.S Department of Health and Human Services, Establishment Registration \& Device Listing, Vol. 2013 (U.S. Food and Drug Administration, 2013).

24. Castro, C.I. \& Briceno, J.C. Perfluorocarbon-Based Oxygen Carriers: Review of Products and Trials. Artif. Organs 34, 622-634 (2010).

25. ClinicalTrials.gov, Vol. 2013 (U.S. National Library of Medicine, 2013). 
26. Kim, P., Kreder, M.J., Alvarenga, J. \& Aizenberg, J. Hierarchical or Not? Effect of the Length Scale and Hierarchy of the Surface Roughness on Omniphobicity of Lubricant-Infused Substrates. Nano Lett. 13, 1793-1799 (2013).

27. Vogel, N. et al. Transparency and damage tolerance of patternable omniphobic lubricated surfaces based on inverse colloidal monolayers. Nat Commun 4, 1-10 (2013).

28. Stark, A.Y. et al. Surface wettability plays a significant role in gecko adhesion underwater. Proc. Natl. Acad. Sci. 110, 6340 - 6345 (2013).

29. Nilsson, J. et al. A randomized study of coronary artery bypass surgery performed with the Resting Heart ${ }^{\mathrm{TM}}$ System utilizing a low vs a standard dosage of heparin. Interactive CardioVascular and Thoracic Surgery 15, 834-839 (2012).

30. Gould, S.A. et al. Fluosol-DA as a Red-Cell Substitute in Acute Anemia. N. Engl. J. Med. 314, 1653-1656 (1986).

31. Epstein, A.K. et al. Liquid-infused structured surfaces with exceptional anti-biofouling performance. Proc. Natl. Acad. Sci. U S A 109, 1318213187 (2012).

32. Tevaearai HT et al. Trillium coating of cardiopulmonary bypass circuits improves biocompatibility. Int. J. Artif. Organs 22, 629 - 634 (1999).

33. Nilsson, L. et al. Heparin-Coated Equipment Reduces Complement Activation during Cardiopulmonary Bypass in the Pig. Artif. Organs 14, 4648 (1990). 
34. Hartmann, R.C. \& Conley, C.L. Studies on the initiation of blood coagulation. III. The clotting properties of canine platelet-free plasma. $J$ Clin Invest. 31, 685 - 691 (1952).

35. Amarnath, L.P., Srinivas, A. \& Ramamurthi, A. In vitro hemocompatibility testing of UV-modified hyaluronan hydrogels. Biomaterials 27, $1416-1424$ (2006).

36. Liu, F. \& Grainger, D.W. in Comprehensive Biomaterials (ed. P. Ducheyne) (Elsevier Science, Oxford, UK; 2011).

37. Toes, G.J. et al. Superhydrophobic modification fails to improve the performance of small diameter expanded polytetrafluoroethylene vascular grafts. Biomaterials 23, 255-262 (2002).

38. Schröder, K. et al. Plasma-Induced Surface Functionalization of Polymeric Biomaterials in Ammonia Plasma. Contrib. Plasma Phys. 41, 562-572 (2001).

39. Canaud, B. et al. Pathochemical Toxicity of Perfluorocarbon-5070, a Liquid Test Performance Fluid Previously Used in Dialyzer Manufacturing, Confirmed in Animal Experiment. J Am Soc Nephrol. 16, 1819 - 1823 (2005).

40. Strobel, M., Lyons, C.S. \& Mittal, K. Plasma surface modification of polymers: relevance to adhesion, Edn. 1. (Vsp, Leiden Netherlands; 1994).

41. Ratner, B.D. Surface modification of polymers: chemical, biological and surface analytical challenges. Biosens. Bioelectron. 10, 797-804 (1995). 
42. Zhang, Z. et al. Polybetaine modification of PDMS microfluidic devices to resist thrombus formation in whole blood. Lab Chip 13, 1963 - 1968 (2013).

43. 10993-4, I. Biological evaluation of medical devices. Part 4: Selection of tests for interactions with blood. (2002).

44. Goodman, S.L. Sheep, pig, and human platelet-material interactions with model cardiovascular biomaterials. J Biomed Mater Res. 45, 240 - 250 (1999).

45. Audran, M. et al. Determination of perfluorodecalin and perfluoro-Nmethylcyclohexylpiperidine in rat blood by gas chromatography-mass spectrometry. J. Chromatogr. B: Biomed. Sci. Appl. 745, 333-343 (2000).

46. Kang, J.H. et al. An Extracorporeal Blood Cleansing Device for Sepsis Therapy. Nature Medicine - in press. (2014). 
Fig. 1
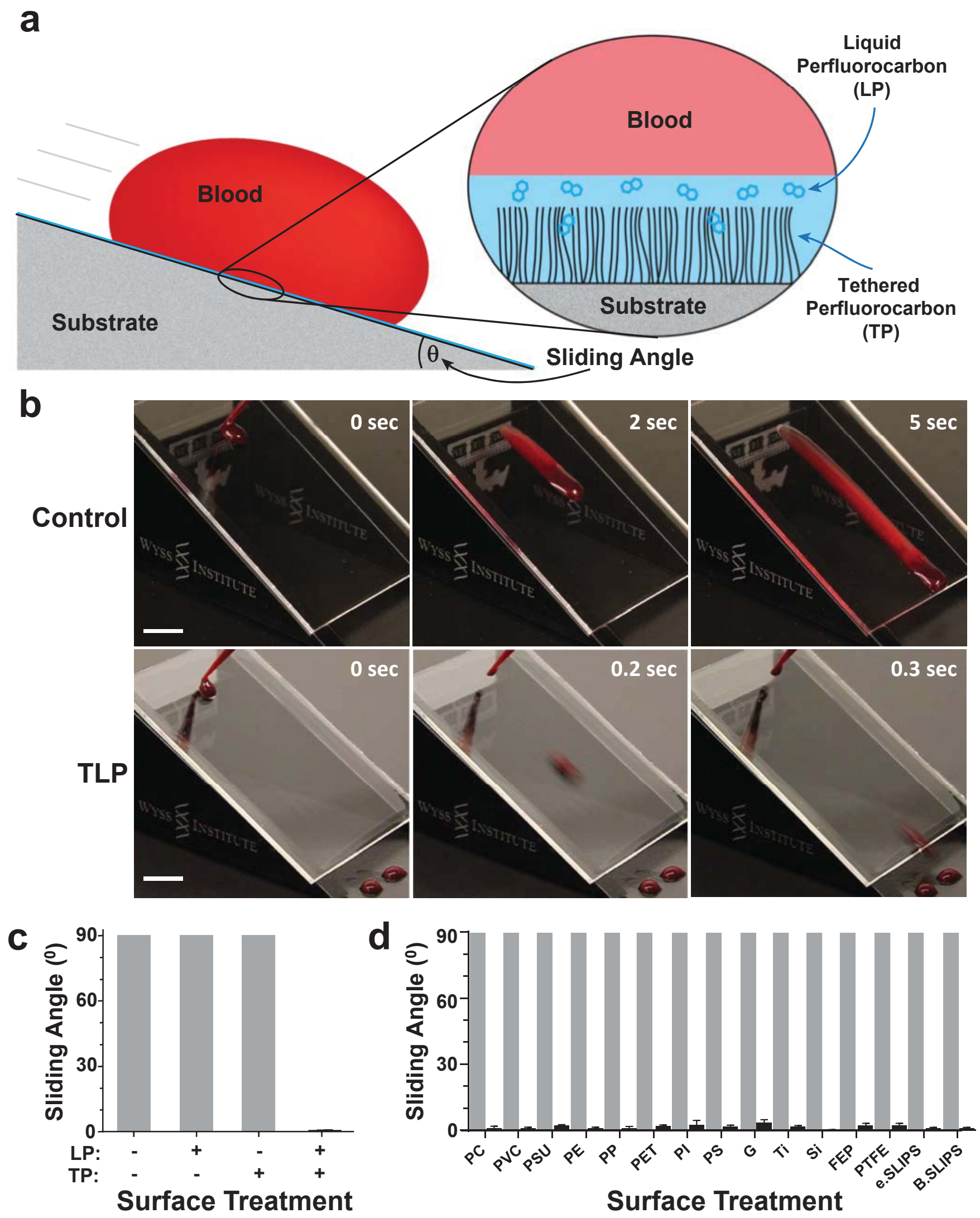
Fig. 2

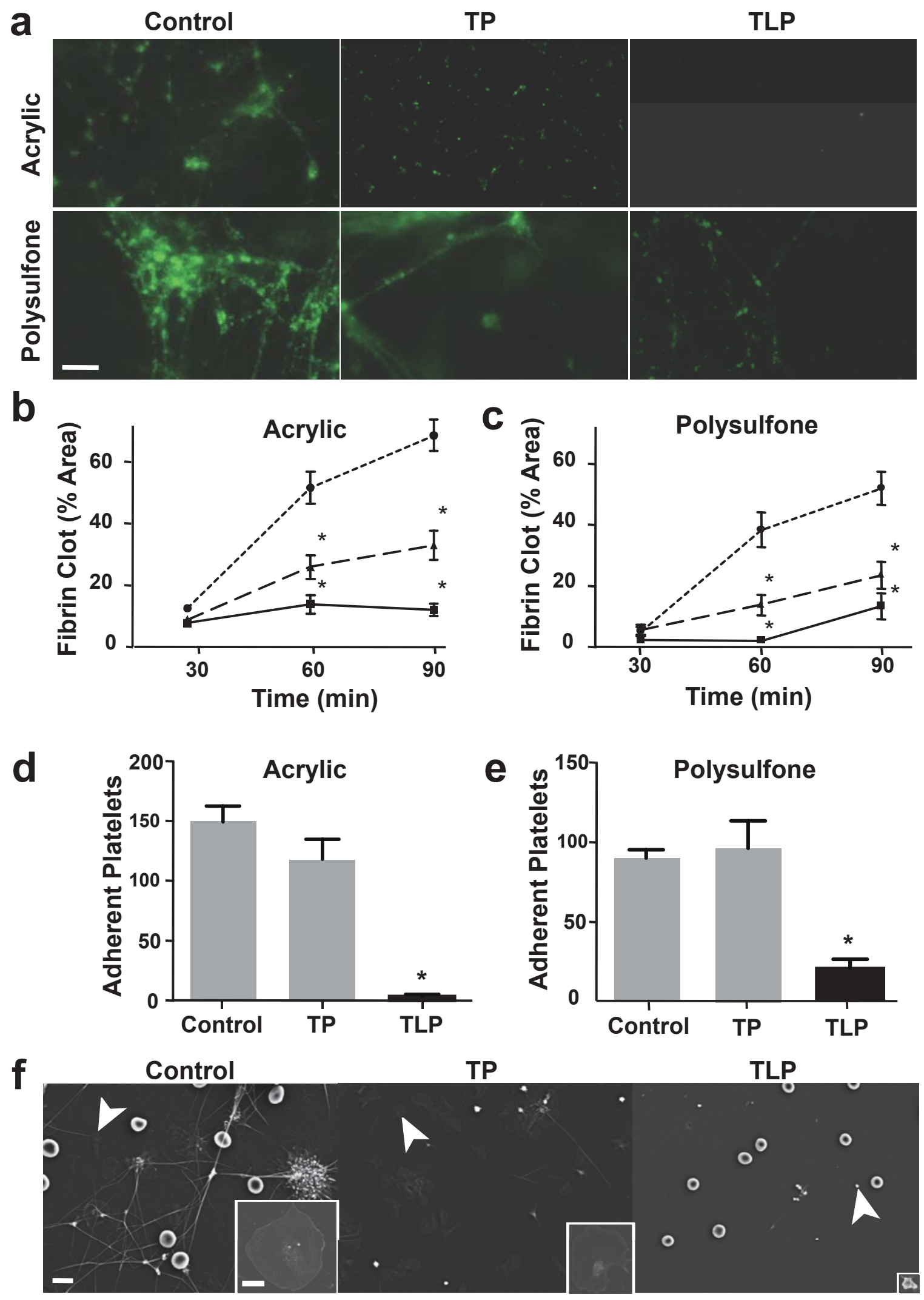


Fig. 3
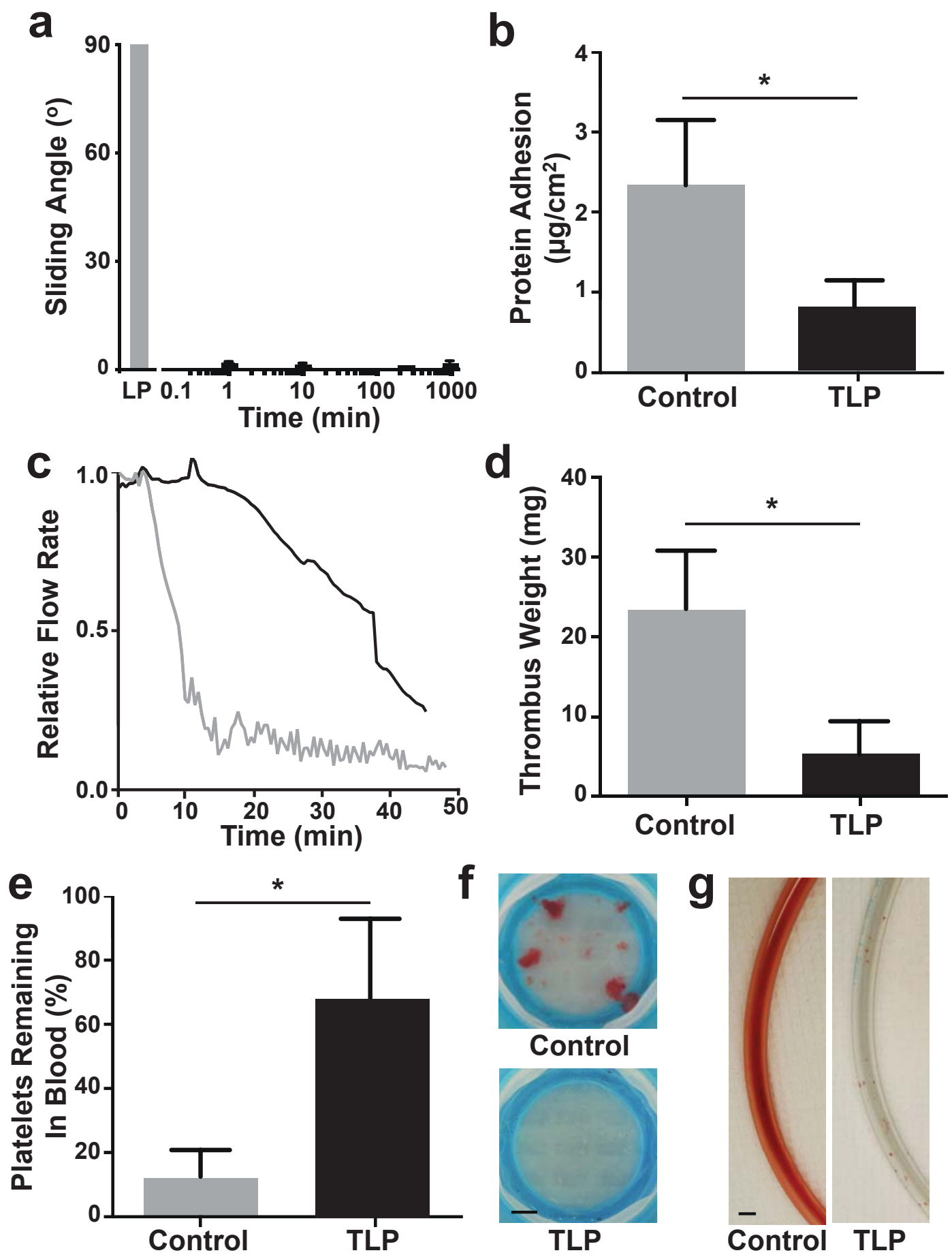
a

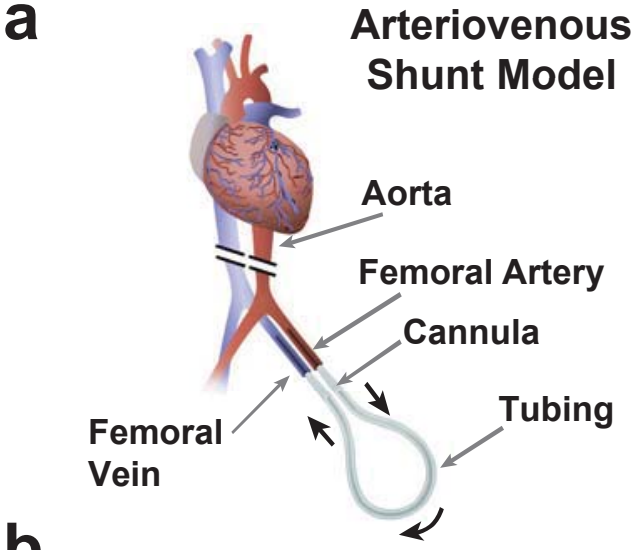

b

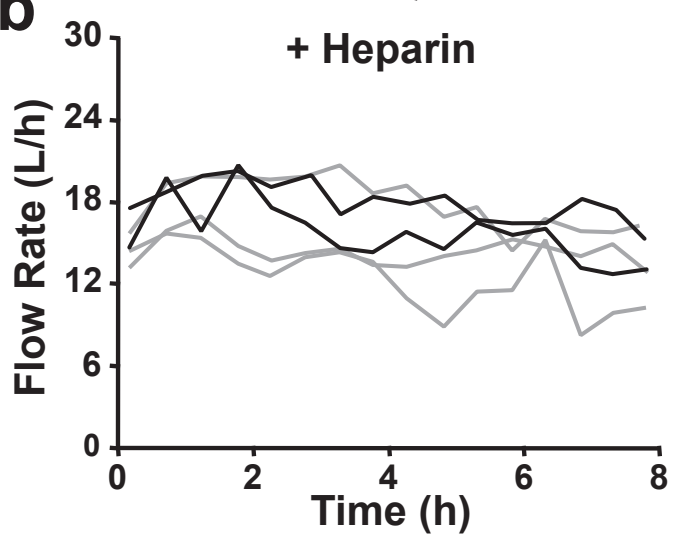

C
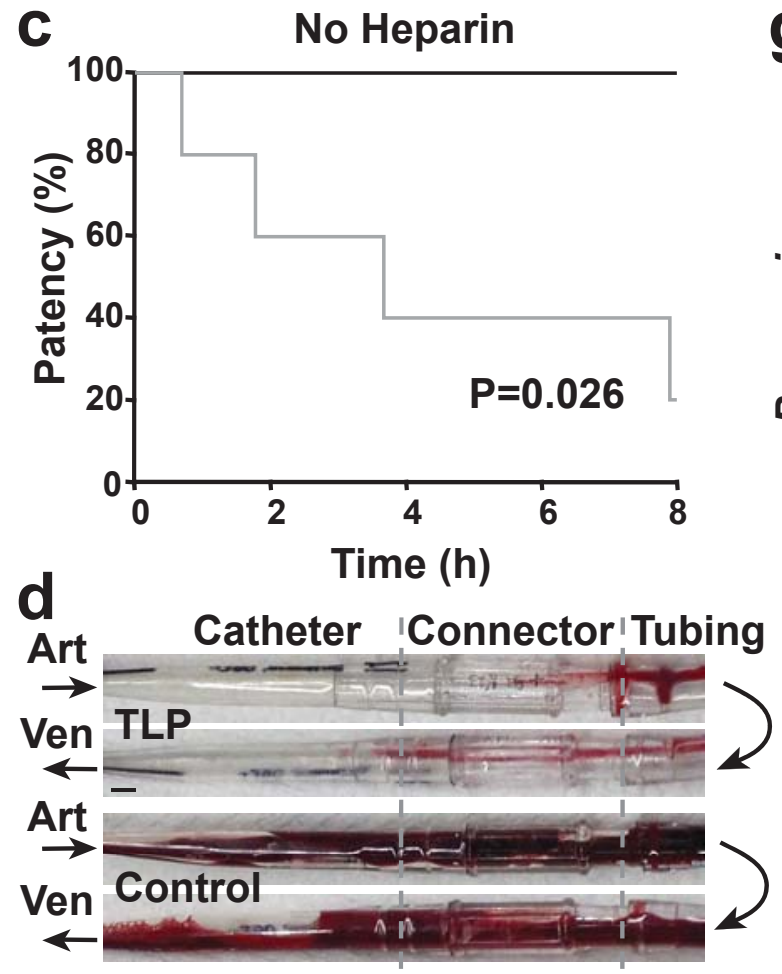

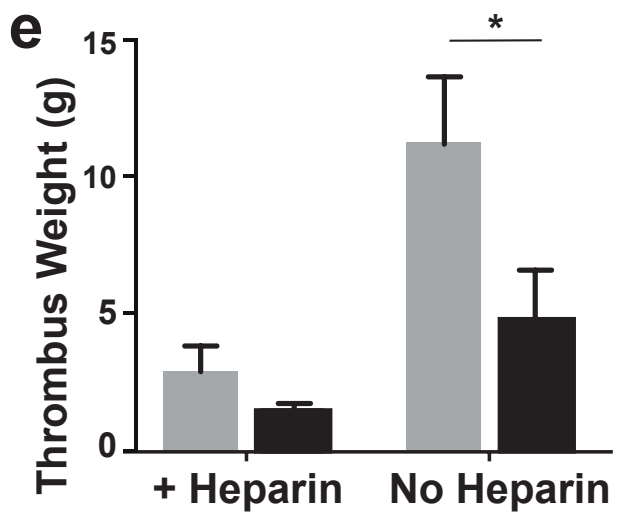

f
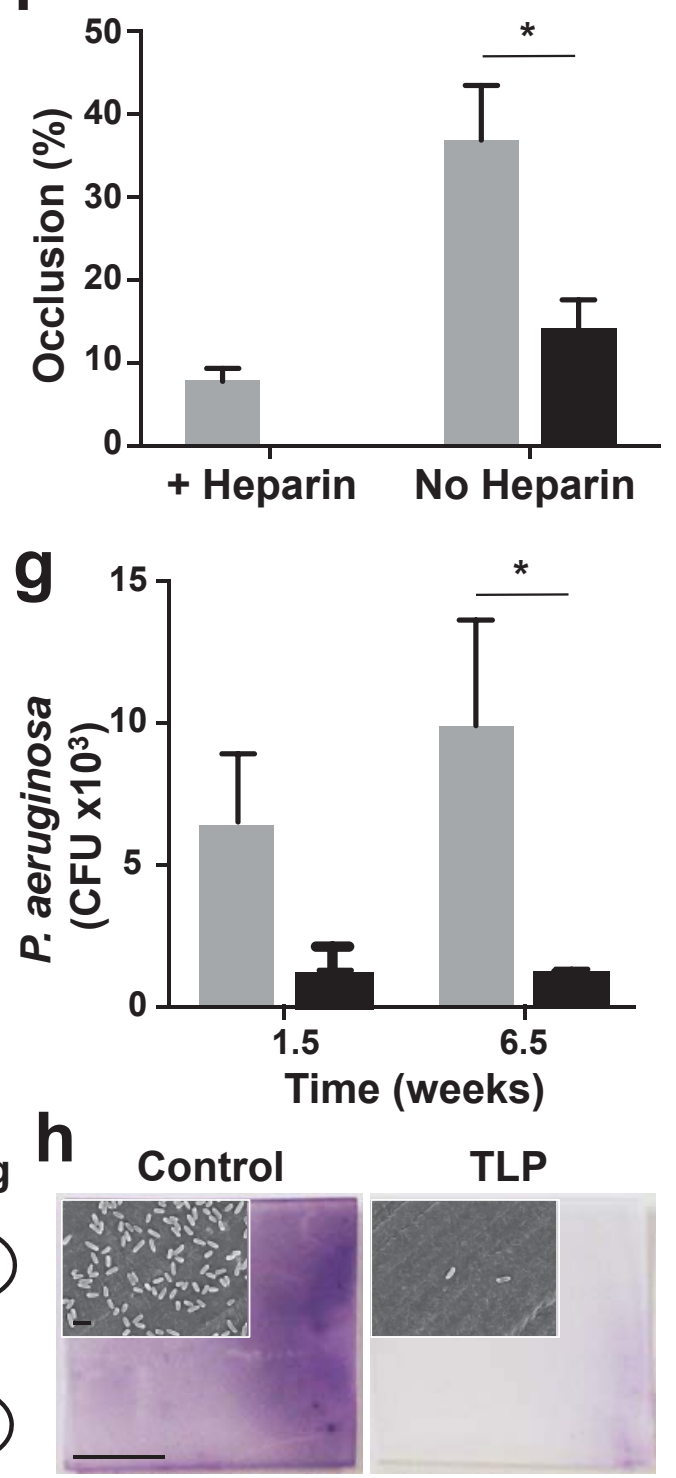

TLP 